\title{
Anti-citrullinated protein antibody and rheumatoid factor in patients with end-stage renal disease
}

\author{
Zeljko Romic ${ }^{1}$, Adriana Unic ${ }^{1, *}$, Lovorka \\ Derek$^{1}$, Marcela Zivkovic ${ }^{1}$, Domagoj \\ Marijancevic ${ }^{1}$, Petar Kes ${ }^{2}$ and Mario Pehar ${ }^{3}$ \\ ${ }^{1}$ Clinical Department for Laboratory Diagnostic, \\ Dubrava University Hospital, Zagreb, Croatia \\ 2 Department of Dialysis, University Hospital Center \\ Zagreb, Zagreb, Croatia \\ ${ }^{3}$ Department of Dialysis, Dubrava University \\ Hospital, Zagreb, Croatia
}

\begin{abstract}
Background: Patients with end-stage renal disease (ESRD) and on hemodialysis (HD) are at increased risk for developing rheumatoid arthritis (RA), as a result of defective immunity. Our aim was to examine if ESRD and the length of HD treatment impact the clinical utility of antibodies to cyclic citrullinated peptides (anti-CCP) and rheumatoid factor (RF) as diagnostic tools for RA.

Methods: We included 94 subjects in our study: 37 healthy volunteers and 57 patients with ESRD who had been undergoing HD for 1-12 years, and without confirmed RA. In order to test our hypothesis, we measured and correlated anti-CCP and RF as laboratory markers of RA.

Results: Our study showed that there is no significant difference between values for anti-CCP $(p=0.11)$ and $\operatorname{RF}(p=0.98)$ in control subjects as well as in patients undergoing $H D$, regardless of the length of time that patients had been undergoing HD treatment.

Conclusions: Our study indicates that HD does not impair the specificity of anti-CCP and RF for RA in patients where the disease has not yet developed. Future prospective studies may show whether there is any use in determinating RF, and especially anti$\mathrm{CCP}$, as early predictors of RA in patients with ESRD who are at greater risk of developing this condition. Clin Chem Lab Med 2009;47:959-62.
\end{abstract}

Keywords: antibodies to cyclic citrullinated peptides (anti-CCP); end-stage renal disease (ESRD); hemodialysis (HD); rheumatoid arthritis (RA); rheumatoid factor (RF).

\section{Introduction}

Hemodialysis (HD) as a treatment for end-stage renal disease (ESRD) may ameloriate the primary disorder,

*Corresponding author: Adriana Unic, BSc in Medical Biochemistry, Department of Laboratory Diagnostics, Dubrava University Hospital, Avenija Gojka Šuška 6, HR-10 000 Zagreb, Croatia

Phone: +385-1-290-3356, E-mail: aunic@kbd.hr Received February 16, 2009; accepted May 6, 2009 but it also leads to the development of new ones, especially in bones, joints and soft tissues (1).

Rheumatoid arthritis (RA), a systemic autoimmune disorder of unknown ethiology causing chronic joint inflammation, affects nearly $1 \%$ of the adult population (2). Patients with ESRD on HD are at increased risk for development of this condition. This risk is due to decreased cellular and humoral immunity, and the requirement for invasive procedures related to urinary and vascular access which promotes bacteremia (3). The 1987 American college of rheumatology (ACR) criteria for RA includes: arthritis of three or more joint areas and positive findings on hand radiographs; arthritis of three or more joint areas including metacarpophageal (MCP) and wrist joints; serum rheumatoid factor (RF)-negative and negative findings on hand radiographs; arthritis involving a single joint area symmetrically and serum RF-positive; oligoarthritis of MCP or wrist joints and serum RF-positive. Classification of RA is very important as new therapies can halt the disease in its early stages. Antibodies to cyclic citrullinated peptides (anti-CCP) are widely used for diagnosis of RA, but are not part of the 1987 ACR classification criteria (4). Anti-CCP antibodies are highly specific for RA (95\%-98\%), and may predict the development of RA before occurrence of the first symptoms of the disease, as well as being good indicators or prognosis $(2,5,6)$. Anti-CCP antibodies are more specific than RF for diagnosing RA, and are better predictive factors for an erosive course of disease (7).

$\mathrm{RF}$ are antibodies directed against antigenic determinants of the Fc portion of $\operatorname{lgG}$, and are increased in $70 \%-80 \%$ of patients with RA. They are also increased in $5 \%-10 \%$ of healthy individuals (8).

Previous studies have shown in patients on HD high levels of circulating immunocomplexes $(9,10)$ that can result in immunological abnormalities and immunosuppressive co-morbidities (3). Our goal was to determine if ESRD and the length of HD impact the utility of anti-CCP and RF as diagnostic markers for RA. We also examined the relationship of these parameters. To test this hypothesis, we measured anti$\mathrm{CCP}$ and RF, comparing patients with ESRD being treated with HD to a healthy control group.

\section{Subjects, materials and methods}

\section{Subjects}

The study was performed at the Clinical Department for Laboratory Diagnostic at University Hospital Dubrava in Zagreb from February to April 2008. The study was approved by the Ethics Committee of University Hospital Dubrava. We included 94 subjects in the study: 37 healthy volunteers in our control group and 57 patients with ESRD who had been 
undergoing HD from 1 year to 12 years. One of the patients in the studied group had been treated with continuous ambulatory peritoneal dialysis (CAPD). The frequency of HD was three times a week for $4 \mathrm{~h}(\mathrm{n}=48)$, three times a week for three and a half hours $(n=6)$ and two times a week for three and a half hours $(n=2)$. The etiologies of ESRD were: hypertension ( $n=19)$, glomerulonephritis $(n=13)$, Balkan endemic nephropathy $(n=6)$, diabetes mellitus $(n=5)$, polycystic kidney disease $(n=4)$, pyelonephritis $(n=3)$, reflux $(n=2)$, kidney stones $(n=2)$, glomerulonephritis combined with hypertension $(n=1)$, drug induced renal failure $(n=1)$ and hereditary kidney disease $(n=1)$.

Demographics of the participants and the presence of bone erosion as assessed by computed tomography, are shown in Table 1. Five patients had no data available concerning the presence of bone erosion.

Patients with ESRD and on HD were divided into two groups, depending on how long they had been undergoing HD (Table 1). The criteria for ESRD patients participating in the study were as follows: patients treated with HD for at least 12 months; and none of the subjects were diagnosed with RA using common diagnostic procedures.

The criteria for the control group consisting of healthy volunteers were as follows: none of the subjects had clinical signs or symptoms of rheumatoid disease noted during examination.

\section{Materials}

The values of anti-CCP and RF were measured in sera collected from patients and controls.

Sera was obtained following centrifugation at $1006 \times g$ for $15 \mathrm{~min}$ in a $35 \mathrm{R}$ Rotina Hettich centrifuge (Tuttlingen, Germany), and then stored at $-20^{\circ} \mathrm{C}$ until analysis.

\section{Methods}

Anti-CCP was measured using the microparticle enzyme immunoassay (MEIA) method with the Abbott AxSym (Chicago, IL, USA). The principle of the method is reaction of anti-CCP from the sample with micro particles coated with CCP. An antigen-antibody complex is formed on micro particles. Alkaline phosphatase conjugate is then added. After the substrate is added (4-methylumbelliferyl phosphate), alkaline phosphatase conjugate catalyzes the hydrolysis of 4-methylumbelliferyl phosphate, yielding the fluorescent product 4-methylumbellyferon. The intensity of the fluorescence is proportional to the concentration of analyte in the sample. The method was calibrated with six levels of antiCCP calibrators (Abbott Laboratories Inc, Chicago, IL, USA). The manufacturer also provided two commercial control samples containing either anti-CCP positive or anti-CCP negative human plasma in phosphate buffer and showing concentrations of $0.0-3.0 \mathrm{U} / \mathrm{mL}$ for the negative control, and $14.0-34.0 \mathrm{U} / \mathrm{mL}$ for the positive control.
The reference interval for anti-CCP reported by the manufacturer is $<3.0 \mathrm{U} / \mathrm{mL}$.

RF was determined using an immunoturbidimetric method on the Olympus AU2700 analyzer (Tokyo, Japan). The principle of the method is reaction of RF with IgG, which coats latex particles resulting in the formation of insoluble aggregates. The absorbance of the created aggregates is proportional to the concentration of RF in the sample.

The manufacturer provided two commercial control samples [ITA Control seras (Olympus Life Science Research Europa $\mathrm{GmbH}$, Munich, Germany)] containing RF at concentrations of 21.1-31.7 $\mathrm{IU} / \mathrm{mL}$ for ITA 1, and $48.1-72.2 \mathrm{IU} / \mathrm{mL}$ for ITA 2.

The reference interval for RF reported by the manufacturer is $<14 \mathrm{IU} / \mathrm{mL}$.

\section{Statistical analysis}

The Kolmogorov-Smirnov test was used to test for a normal distribution of the data. The $\chi^{2}$-test and one-way analysis of variance (ANOVA) were used for data comparisons. A $\mathrm{p}<0.05$ was considered statistically significant. MedCalc 9.2.0.0 statistical software (MedCalc, Mariakerke, Belgium) was used for statistical analysis.

\section{Results}

Values for anti-CCP and RF in the groups are shown in Table 2.

All numerical parameters were normally distributed (Table 1). Thus, the data were tested using parametric analysis and shown as mean and SD. One-way ANOVA did not show statistically significant differences between values for anti-CCP $(p=0.11)$ and $\operatorname{RF}(p=0.98)$ among groups (control group, N1 and N2), and the values were within the manufacturer's reference interval. Correlation between anti-CCP and RF was not statistically significant.

Results for analysis of quality control material for the anti-CCP negative control, anti-CCP positive control, ITA 1 control serum and ITA 2 control serum were within the manufacturer's recommended ranges.

The frequency of increased values of anti-CCP ( $>3 \mathrm{U} / \mathrm{mL}$ ) were as follows: 0/37 in the control group; $4 / 57$ in all patients with ESRD and on HD (2/32 in the subgroup on HD for $1-5$ years, and $2 / 25$ in the subgroup on $\mathrm{HD}>5$ years).

The frequency of increased values of RF $(>14$ $\mathrm{IU} / \mathrm{mL}$ ) were as follows: $1 / 37$ in the control group; $2 /$ 57 in all patients with ESRD and on HD (1/32 in the subgroup on HD from $1-5$ years, and $1 / 25$ in the subgroup on $\mathrm{HD}>5$ years).

Table 1 Basic characteristics of control group and patients with ESRD on hemodialysis.

\begin{tabular}{lllll}
\hline Parameter & $\begin{array}{l}\text { Control } \\
\text { group }\end{array}$ & $\begin{array}{l}\text { Patients with } \\
\text { ESRD on } \\
\text { hemodialysis }\end{array}$ & $\begin{array}{l}\text { Patients on } \\
\text { hemodialysis } \\
1-5 \text { years N1 }\end{array}$ & $\begin{array}{l}\text { Patients on } \\
\text { hemodialysis } \\
5 \text { years or more N2 }\end{array}$ \\
\hline $\mathrm{n}$ & 37 & 57 & 32 & 25 \\
Age, years (mean \pm SD) & $50 \pm 7$ & $54 \pm 13$ & $54 \pm 13$ & $55 \pm 12$ \\
Sex (male/female) & $17 / 20$ & $31 / 26$ & $18 / 14$ & $13 / 12$ \\
Bone erosions & $0 / 37$ & $19 / 52$ & $8 / 29$ & $11 / 23$ \\
\hline
\end{tabular}

ESRD, end-stage renal disease. 
Table 2 Anti-CCP and RF values of control group and ESRD patients on hemodialysis.

\begin{tabular}{|c|c|c|c|c|c|}
\hline Parameter & Control group & $\begin{array}{l}\text { Patients with } \\
\text { ESRD on } \\
\text { hemodialysis }\end{array}$ & $\begin{array}{l}\text { Patients on } \\
\text { hemodialysis } \\
1-5 \text { years N1 }\end{array}$ & $\begin{array}{l}\text { Patients on } \\
\text { hemodialysis } \\
5 \text { years or more N2 }\end{array}$ & $\mathrm{p}$-Value \\
\hline $\begin{array}{l}\text { Anti-CCP, U/mL } \\
\text { (mean } \pm \text { SD) }\end{array}$ & $1.46 \pm 0.60$ & $1.1 \pm 0.51$ & $1.26 \pm 0.51$ & $1.17 \pm 0.51$ & 0.11 \\
\hline $\begin{array}{l}\mathrm{RF}, \mathrm{IU} / \mathrm{mL} \\
(\mathrm{mean} \pm \mathrm{SD})\end{array}$ & $6.38 \pm 3.03$ & $6.43 \pm 5.03$ & $6.32 \pm 6.05$ & $6.57 \pm 3.42$ & 0.98 \\
\hline
\end{tabular}

ESRD, end-stage renal disease; anti-CCP, antibodies to cyclic citrullinated peptides; RF, rheumatoid factor.

\section{Discussion}

ESRD affects all body systems due to the significant decrease in renal function. Changes in bones, joints and soft tissues occur often $(1,3,9,10)$. This is particularly important for RA which is associated with significant morbidity and mortality. It is important to recognize this disease as soon as possible, in order to implement therapy promptly and prevent further deterioration. HD is the most common procedure used for treating advanced and permanent renal failure. While the HD procedure has advanced significantly and become simpler and more efficient, there are still numerous adverse effects. Our patients were divided into two groups based on years of treatment with HD. We used a limit of 5 years of HD since it is known that there may be signs of amyloidosis (dialysis-related amyloidosis) following five or more years of HD. Amyloidosis develops when proteins in the blood form deposits in joints and tendons, causing pain and joint stiffness, as is the case with arthritis (11). Patients on long-term peritoneal dialysis have less disorders and prolonged residual renal function (RRF) following 5 years of therapy (12). However, our study included only one patient on long-term peritoneal dialysis.

Previous studies reported a higher incidence of septic arthritis (3) and autoimmune complexes $(9,10)$ in patients on HD. As anti-CCP is an early predictor of RA $(13,14)$, and RF is the only diagnostic laboratory test for diagnosis of RA (4), we wanted to determine whether ESRD and HD treatment, as well as the length of HD, affects the values of these parameters. The high prevalence of autoimmune complexes in hemodialyzed patients $(9,10)$ could point to the development of autoimmune disorders. Thus, anti-CCP and RF could be useful diagnostic tools for monitoring the development of disease (15-17). Also, it is possible that the HD procedure removes immune complexes as well as the other auto-antibodies such as anti-CCP and RF (18). This would explain the reason why we did not find a statistically significant difference associated with length of HD.

Our results showed that anti-CCP and RF concentrations were not changed in patients with ESRD who were on HD, as well as in the subgroups depending on length of HD. These finding indicate that HD does not impair the specificity of anti-CCP and RF measurements. Also, it is possible that there is no value in measuring the concentration of these two analytes for early detection of RA in patients with ESRD. However, this should be investigated by prospective monitoring of patients. Previous studies have shown that antiCCP is an early predictor of RA, and that concentrations correlate with disease severity (14). Our results suggest that HD is not likely to be the trigger for development of RA.

Since our results did not show increased values of anti-CCP and RF, antibodies that are present in high concentrations in patients with ESRD treated with HD should be clearly identified. Future prospective studies may determine if there is any use in determinating $\mathrm{RF}$, and especially anti-CCP, as early predictors of RA in ESRD patients who might be at greater risk of developing this condition.

\section{References}

1. Grennan DM, McMahon MJ. Renal disease and rheumatic manifestations. Curr Opin Rheumatol 1993;5:77-8.

2. Khosla P, Shankar S, Lalit D. Anti CCP antibodies in rheumatoid arthritis. J Indian Rheumatol Assoc 2004:12: $143-4$.

3. Al-Nammari SS, Gulati V, Patel R, Bejjanki N, Wright M. Septic arthritis in haemodialysis patients: a seven-year multi-centre review. J Orthop Surg (Hong Kong) 2008;16: 54-7.

4. Liao KP, Batra KL, Chibnik L, Schur PH, Costenbader KH. Anti-CCP revised criteria for the classification of rheumatoid arthritis. Ann Rheum Dis 2008;67:1557-61.

5. Nishimura K, Sugiyama D, Kogata Y, Tsuji G, Nakazawa $T$, Kawano S, et al. Meta-analysis: diagnostic accuracy of anti-cyclic citrullinated peptide antibody and rheumatoid factor for rheumatoid arthritis. Ann Intern Med 2007; 146:797-808.

6. Herold M, Boeser V, Russe E, Klotz W. Anti-CCP: history and its usefulness. Clin Dev Immunol 2005;12:131-5.

7. Silveira I, Burlingame R, Mühlen C, Bender A, Staub H. Anti-CCP antibodies have more diagnostic impact than rheumatoid factor (RF) in a population tested for RF. Clin Rheumatol 2007;26:1883-9.

8. Wilson D. Rheumatoid factors in patients with rheumatoid arthritis. Can Fam Physician 2006;52:180-1.

9. Perez GO, Glasson P, Favre H, Wauters JP, Benzonana $G$, Jeannet $M$, et al. Circulating immune complexes in regularly dialyzed patients with chronic renal failure. Am J Nephrol 1984;4:215-21.

10. Gagnon RF, Shuster J, Kaye M. Auto-immunity in patients with end-stage renal disease maintained on hemodialysis and continuous ambulatory peritoneal dialysis. J Clin Lab Immunol 1983;11:155-8.

11. Tran M, Rutecki GW, Spraque SM. The pathogenesis of beta2-microglobulin-induced bone lesions in dialysisrelated amyloidosis. Semin Dial 2001;14:131-3. 
12. Davies SJ, Phillips L, Girffiths AM, Russell LH, Naish PF, Russell GI. What, really happens to people on long-term peritoneal dialysis? Kidney Int 1998;54:2207-17.

13. Kim KE, Kim KH, Woo KS, Han JY, Kim JM, Lee SW, et al. Diagnostic utility of AxSYM anti-cyclic citrullinated peptide antibody assay. Korean J Lab Med 2008;28: 457-64.

14. Berglin E, Johansson T, Sundin U, Jidell E, Wadell G, Hallmans G, et al. Radiological outcome in rheumatoid arthritis is predicted by presence of antibodies against cyclic citrullinated peptide before and at disease onset, and by IgA-RF at disease onset. Ann Rheum Dis 2006; 65:453-8.
15. Naik $\mathrm{S}$. The immunological basis for autoimmunity. $J$ Indian Rheumatol Assoc 2004:12;22-8.

16. Newkirk MM. Rheumatoid factors: host resistance or autoimmunity? Clin Immunol 2002;104:1-13.

17. Nielen MM, van Schaardenburg D, Reesink HW, van de Stadt RJ, van der Horst-Bruinsma IE, de Koning $\mathrm{MH}$, et al. Specific autoantibodies precede the symptoms of rheumatoid arthritis: a study of serial measurements in blood donors. Arthritis Rheum 2004;50:380-6.

18. Inagi R, Miyata T, Hong K, Pramoonjago P, Maeda K, Inoue K. Decreased activity of complement-mediated immune complex clearance in hemodialysis patients. Clin Immunol Immunopathol 1993;68:333-9. 